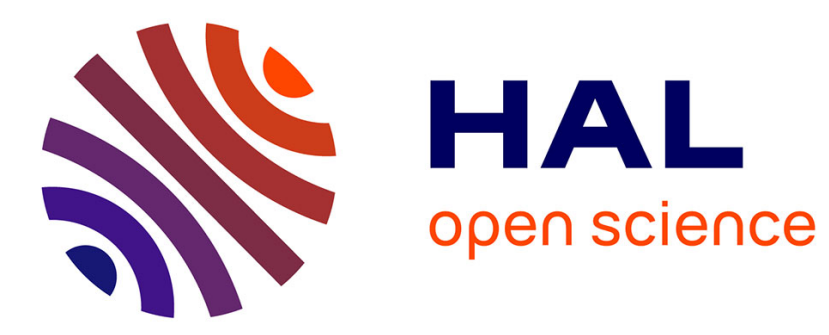

\title{
The Curious Strategy of Multipartite Viruses
}

Yannis Michalakis, Stéphane Blanc

\section{To cite this version:}

Yannis Michalakis, Stéphane Blanc. The Curious Strategy of Multipartite Viruses. Annual Review of Virology, 2020, 7 (1), pp.203-218. 10.1146/annurev-virology-010220-063346 . hal-02981664

\section{HAL Id: hal-02981664 https://hal.inrae.fr/hal-02981664}

Submitted on 25 Nov 2020

HAL is a multi-disciplinary open access archive for the deposit and dissemination of scientific research documents, whether they are published or not. The documents may come from teaching and research institutions in France or abroad, or from public or private research centers.
L'archive ouverte pluridisciplinaire HAL, est destinée au dépôt et à la diffusion de documents scientifiques de niveau recherche, publiés ou non, émanant des établissements d'enseignement et de recherche français ou étrangers, des laboratoires publics ou privés. 
1 Title: The Curious Strategy of Multipartite Viruses

2 Authors: Yannis Michalakis ${ }^{1}$ and Stéphane Blanc ${ }^{2}$

3

4 Affiliations:

$5 \quad{ }^{1}$ MIVEGEC, CNRS, IRD, Univ Montpellier, Montpellier, France

$6{ }^{2}$ UMR BGPI, INRA, CIRAD, Montpellier SupAgro, Univ. Montpellier, Montpellier, France

7

8

9 ORCID IDs:

10 YM: $0000-0003-1929-0848$

11 SB: 0000-0002-3412-0989

12

13 Correspondence should be sent to both YM and SB:

$14 \quad \underline{Y a n n i s . M i c h a l a k i s @ i r d . f r ~}$

15 Stephane.blanc@inra.fr

16

17 
Keywords : multipartite virus, genome architecture, virus evolution, multicomponent virus, genome formula, multicellular functioning.

Abstract : Multipartite virus genomes are composed of several segments, each packaged in a distinct viral particle. While this puzzling genome architecture is found in $\sim 17 \%$ of known viral species, its distribution among hosts or among distinct types of genome composing nucleic acid remain poorly understood. No convincing advantage of multipartitism has been identified, yet the maintenance of genomic integrity appears problematic. We here review recent studies shedding light on these issues. Multipartite viruses rapidly modify the copy number of each segment/gene from one host species to another, a putative benefit if host switches are common. One multipartite virus functions in a multicellular way: the segments do not need to all be present in the same cell and can functionally complement across cells maintaining genome integrity within hosts. The genomic integrity maintenance during host-to-host transmission needs further elucidation. These features challenge several Virology foundations and could apply to other multicomponent viral systems. 


\section{WHAT ARE MULTIPARTITE VIRUSES - DISTINCTION WITH MONOPARTITE/SEGMENTED}

Viruses show exceptional variation in how they package their genetic information for transmission to future generations. Most viruses have their genetic information carried by a single molecule of DNA or RNA packaged in a transmission vehicle, the viral particle. These are the monopartite viruses such as the dsDNA herpesviruses, the ssDNA geminiviruses (with the exception of some begomoviruses) or parvoviruses, the dsRNA totiviruses, the (+)ssRNA polioviruses and the (-)ssRNA filoviruses, and they represent $\sim 75 \%$ of all viral annotated species (1). Another strategy occurs only among RNA viruses and consists in having the genetic information carried by several molecules which are all packaged together in a single viral particle. These are the segmented viruses, which despite their relative scarcity, representing only $\sim 9 \%$ of current viral species (1), are well known probably because they comprise some famous human parasites such as the Influenza A Virus. A third, puzzling, strategy goes one step further: the genetic information is carried by several molecules, but instead of packaging all these molecules together, these viruses package them in different viral particles. These are the multipartite viruses. Despite the fact that they represent $\sim 17 \%$ of all viral species, their study has been widely neglected and their Biology remains largely mysterious. They recently attracted renewed interest, as two recent reviews $(1,2)$ illustrate. In this paper we rapidly recall some important points and review important recent discoveries (Figure 1). We then provide arguments on how these recent findings on multipartite viruses challenge some Virology foundations, call for a reconsideration of some important notions in Biology, and invite us to reconsider a number of features of other viral multicomponent systems.

\section{DISTRIBUTION OF MULTIPARTITISM IN THE VIRAL WORLD:}


Multipartite viruses are very common parasites of plants and fungi. Indeed, about a

third of viral genera parasitizing plants have adopted this peculiar genomic organization.

Because they cause disease in many agriculturally important plants, multipartite viruses have important financial and well-being consequences (2).

The host distribution of multipartite viruses is intriguing (Figure 2). Indeed, none has ever been reported to infect bacteria, while the reports in animals can be counted with the fingers of just one hand and are of two sorts. The first sort corresponds to plant viruses, e.g. the genus Tenuivirus (3), which also replicate in their arthropod vectors. The genus Dichorhavirus (Family Rhabdoviridae) may also be included in this category (4), although both the replication within the mite vector (5) and the actual separate encapsidation of the two genome segments await definitive confirmation. The second sort regroups viruses exclusively infecting animal hosts. The separate encapsidation of the genome segments has been definitely confirmed solely for bidensoviruses of silkworms (6). One additional case, a Jingmenvirus infecting mosquitoes (Guaico culex virus, GCXV), has recently been proposed (7). To infer that GCXV is multipartite, this study used dilution of virus particles-containing solutions and titer monitoring, as well as infection of cell cultures at low MOI demonstrating that the distinct particles infecting different cells do not contain the full set of segments. However, a similar low MOI-cell culture infection has been used in a segmented bunyavirus to demonstrate that most viral particles do not contain all the genomic segments due to their non-specific encapsidation (8). Therefore further studies in Jingmenviruses are needed to conclusively discard alternative genomic characterizations. 
In fact, the formal qualification of a viral species as "multipartite" cannot derive from sequence comparisons and homology because there are numerous examples of monopartite species highly homologous to multipartite ones, belonging to the same viral families or even genera (e.g. the family Potyviridae or the genus Begomovirus). It can also not be derived solely from the dilution techniques mentioned above. Despite their usefulness to originally conceive the existence of multipartite viruses of plants (9), it is now clear that serial dilutions of virus suspensions could similarly affect the titration (concentration of infectious units) for both multipartite and segmented viruses producing a large amount of semi-infectious particles (8, 10). Consequently, approaches directly assessing the number and nature of segments encapsidated in distinct particles within a virus population are required. This can be achieved by the physical separation of distinct classes of virus particles from a population and analysis of their respective genome content $(11,12)$. But techniques of structural biochemistry $(13,14)$ or fluorescent high-resolution microscopy $(8,15)$ are also efficient.

Current data indicate that multipartite viruses appear common in plants and fungi, extremely rare in animals and inexistent in bacteria (Figure 2A). The reason for this distribution is at present unclear. Although it is difficult to formally exclude a sampling bias without detailed quantifications of relative sampling effort, this explanation is not very plausible: for example, there is no reason to expect that the viromes of animal farms have been investigated in a more biased way than those of crops, nor is there a reason to see why phage investigations would have missed all multipartite species if they existed. The distribution is so skewed, that a few hypothetical missed cases would not balance it, and thus it likely represents a biological reality. What may be the reasons underlying it? 

studies were based, understandably, on the then knowledge, or beliefs. It was then believed that plant viruses had high multiplicities of infection, which could avoid or at least limit the risk of losing genomic integrity upon cell-to-cell or host-to-host transmission. Zwart and Elena (21) reviewed empirical estimations of the multiplicities of infection at the individual cell, withinhost across organ, and the between host levels and the existing evidence does not indicate that plant viruses, independently of their genomic architecture, stand out in any way (see later section for more discussion on this issue). A recent explanation put forward by Zhang et al. (22) is that, under some assumptions, multipartite viruses require lower endemic thresholds to be maintained in hosts connected by dense static networks, i.e. networks with numerous neighbor contacts which do not change over time, than in annealed networks where neighbor contacts are reshuffled continuously. Zhang et al. concluded that this result explains why multipartite viruses occur mostly in plants and almost never in animals, because static networks would better represent plant contact networks while annealed networks would better represent animal contact networks. There are several issues with this proposed explanation but the most important is that horizontally transmitted plant multipartite viruses are vectored by animals, depends mostly on animal movements and whatever motivates them. The reason underlying the host distribution of multipartite viruses thus still eludes us, and it is not even clear whether we should expect an ecological or a cellular/physiological type of explanation.

Another intriguing aspect of the distribution of multipartite viruses concerns the nucleic acid supporting their genomic information (Figure $2 B$ and $(1,2,23))$. One can note that: 
- dsDNA viruses are exclusively monopartite; their genome is never segmented nor multipartite

122 (we exclude polydnaviruses for reasons discussed in (2)

- ssDNA viruses are either mono- or multipartite; there are no segmented ssDNA viruses

- (+)ssRNA viruses are mostly mono- or multipartite, only nodaviruses and omegatetravirus being segmented.

- (-)ssRNA viruses are mostly monopartite or segmented, with the exception of the genera

- dsRNA viruses are the only case where monopartite are a minority; most of them are segmented, with roughly equal numbers of genera among multipartite and monopartite. be segmented than multipartite, why are dsRNA viruses so labile and why are dsDNA viruses so intolerant of any sort of genome segmentation? To complicate matters, the reasons underlying host distributions and those underlying nucleic acid distributions could be confounded. For example, only few dsDNA viruses parasitize plants or fungi (Figure 2C) (24). Of the 27 bacteriophage families currently recognized by the ICTV all have dsDNA genomes but four ssDNA and two RNA families (23, Figure $2 \mathrm{C}$ ). Thus for this group of viruses it is difficult to tell whether they do not become multipartite because they rarely infect plants or fungi, or whether they do not become multipartite because there is something in dsDNA that makes it less amenable to multipartitism and segmentation. Each of the remaining nucleic acid genomic 
multipartite-excluding hosts (i.e. animals, bacteria and Archaea) such that within each the relative representation of genomic architectures may at least partially reflect that of the respective hosts.

Probably because their existence is puzzling, it is often assumed, e.g. in theoretical considerations, that multipartite viruses are derived from ancestral monopartite viruses. Plausible as this may be, it has actually never been investigated through e.g. phylogenetic reconstructions. The relationship between multipartite and segmented viruses has also eluded phylogenetic analyses so far: as the previous paragraph discusses these two genomic architectures do not co-occur in some nucleic acid configurations, but they do in other. When they do co-occur, do they co-occur in the same branches, and if yes, which is ancestral? These important questions still await for a rigorous analysis.

\section{PROPOSED ADVANTAGES OF MULTIPARTITE VIRUSES}

The proposed advantages have been already reviewed elsewhere $(1,25)$. We thus only briefly mention them here.

The factors initially put forward, and developed with theoretical models, all derive from the fact that each segment of a multipartite virus is smaller than the entire genome of a monopartite, for a given total genome length. Thus multipartite viruses would have evolved because (i) smaller segments would suffer less from high mutational loads, due to high mutation rates $(16,17)$; (ii) would replicate faster $(17,19,26)$; (iii) because of their segmented nature they would benefit from genetic exchanges (26-28). It is worth noting that none of these hypotheses is specific to multipartite viruses: while the theoretical models developed to 
back them explicitly considered multipartite genomic architectures, the above features are shared with segmented viruses. It is thus not obvious why a multipartite rather than a segmented genomic architecture should be adopted; the latter would not incur the costs specific to multipartitism pertaining to the preservation of viral genomic integrity (see next section). Further, while these putative benefits have unfortunately rarely been tested, the only published test failed to find any evidence in their favor (29): after passaging the monopartite foot and mouth disease virus (FMDV) at high MOI, Garcia-Arriaza and colleagues observed the spontaneous evolution to a bipartite variant (30). Ojosnegros and colleagues subsequently showed that the bipartite outcompeted the monopartite variant, but none of the above cited processes was responsible for this outcome; instead, the viral particles of the bipartite variant were more stable allowing for a longer infectious period (29). The lack of any other cases renders it impossible to know how general this explanation could be, both as to its occurrence and as to the magnitude of the potential benefit for multipartite forms. Finally it was recently shown (31) that even though the evolution of multipartitism is improbable in the absence of an intrinsic advantage, it may rarely occur following the stochastic extinction of monopartite forms under specific parameters allowing for the maintenance of multipartite variants. This is unlikely as a general explanation of the evolution of multipartitism, but should not be ignored for explaining specific cases.

\section{POTENTIAL COSTS AND HOW MULTIPARTITE VIRUSES SOLVE THEM:}

The existence of multipartite viruses appeared problematic since their discovery because of the potential issue of the maintenance of their genomic integrity: if all segments need to be present in the same host individual and, presumably, individual host cell for the 
infection to function, by packaging the segments separately multipartite viruses potentially incur the risk of producing many incomplete non-functional inoculations.

It is a priori possible to imagine two potential ways through which this transmission cost could be resolved (Figure 1): (i) through independent transmission of a sufficiently large number of viral particles to ensure that at least one copy of each is transmitted at the relevant level (individual host for between-host transmission, Figure 1.5, or individual cell for withinhost transmission, Figure 1.2); or (ii) through some sorting mechanism that manages to bring together at least one copy of each segment and achieve their sorted transmission even if the total number of transmitted segments is small. Either of these mechanisms could apply at either level. The potential of the first mechanism to resolve this cost has been evaluated theoretically by Iranzo and Manrubia (32), who calculated the number of viral particles that would need to be transmitted, designated by the authors as MOI for multiplicity of infection, in order for a multipartite variant to outcompete a monopartite ancestor. These calculations showed that while for multipartite viruses with few segments the threshold MOIs are not too high, for viruses with more than four segments they are orders of magnitudes larger than the currently available empirical MOI estimates for any kind of virus.

\subsection{Within-host cost}

\subsubsection{Genome formulas}

A first study formally questioned whether the eight segments of the octopartite faba bean necrotic stunt virus (FBSNV, genus Nanovirus, family Nanoviridae), which code for one gene each and are all approximately $1 \mathrm{~kb}$ long, accumulate within individual plants at equal 
frequencies. The answer was that they do not: some segments accumulate at low frequencies while others are much more frequent (33). Infections converge to a sort of 'equilibrium' frequency distribution, termed the 'genome formula', as the disease progresses within plants. When the virus is transmitted from one host plant species to another, the genome formula changes within a single passage. Similar observations have since been reported in another plant multipartite virus, the (+)ssRNA tripartite alfalfa mosaic virus (AMV (34)). The existence of an uneven genome formula has also been concluded for one of the rare multipartite viruses infecting an animal, the ssDNA bipartite bombyx mori bidensovirus ( $\mathrm{BmBDV}(35)$ ), although a single host was tested in this latter case. It is also possible to reinterpret the results of previous studies as indicative of the existence of a genome formula in other viruses (see the end of the Discussion of (33) for references), suggesting that the unequal accumulation of the distinct genome segments is a general feature during infection of hosts by multipartite viruses.

The existence of the formula adds to the cost: rare segments would require an even larger $\mathrm{MOI}$ to be transmitted than that corresponding to all segments having equal frequencies. Indeed, Sofonea and colleagues (appendix of (36)) extended the calculations of Iranzo and Manrubia (32) and showed that when accounting for the existence of the genome formula the threshold MOIs beyond which multipartitism may evolve become even larger.

On the other side, the fact that the genome formula readily changes when the viruses are passaged from one host species to another suggests that the multipartite nature of the genome of these viruses allows them to rapidly modify the relative expression of their genes (Figure 1.4). The fact that variation in segment copy numbers has functional consequences was recently investigated by Gallet and colleagues (R. Gallet, J. Di Mattia, S. Ravel, R. Vitalis, Y. 
Michalakis and S. Blanc in preparation): they found that the amount of DNA of each segment is positively correlated to the amount of its corresponding mRNA in two host plant species. This result shows that the genome formula variation indeed impacts gene expression. Whether this variation is adaptive remains to be formally demonstrated empirically; the possibility that it may represent an adaptive strategy in a rapidly changing environment, such as an environment where a virus changes host species frequently, has been demonstrated by a recent theoretical study (M. Zwart \& S.F. Elena in review).

\subsubsection{A multicellular way of life}

The fact that in order to avoid the cost of multipartitism viruses would need to have unrealistically high MOIs, at least under the hypothesis that the different segments do not 'travel' in a sorted way, led us to question the premise of the cost at least at the within-host level: is it true that all genomic segments need to be concomitantly present within each host cell for the infection to function? Using fluorescent-labeling techniques we found that the distinct segments of FBNSV do not co-occur in most cells of an infected host (37). This per se could be interpreted as a manifestation of the cost of multipartitism. However, even though the segments often do not co-occur, a given segment often co-occurs in a cell with the protein encoded by a segment which is locally absent. Thus, the function of a segment is present in cells where the genetic information is absent, suggesting that at least FBNSV infections operate at a supra-cellular level through trafficking of gene products; whether the function circulates under the form of mRNA, protein or both, remains to be investigated. The main point, however, is that the genomic segments do not need to be concomitantly present in the same cell for the infection to progress; therefore the putative cost of multipartitism should be much smaller than 
initially anticipated at least at the within-host level (Figure 1.1). A more precise quantification of this putative cost would require identifying the spatial scale at which the function trafficking occurs. If this is the entire plant, then the putative cost at this level could potentially be nil.

\subsubsection{Sorted transmission}

Although not experimentally demonstrated, the possibility that sorted transmission of the distinct genome segments could occur in multipartite viruses has been suggested for some viral species. We use the term 'sorted transmission' here to distinguish it from "collective transmission" as commonly used in the literature. Collective transmission connotes the cotransmission of several virus particles to a cell or a host, and because it is most commonly conceived for monopartite viruses, it usually designates the joint delivery of multiple copies of a viral genome and thus increased $\mathrm{MOI}$ (for review see (38)). The phenomenon we discuss here is that multipartite viruses could sort their distinct genome segments, somehow assembling them to constitute a transmitted group containing the integral genome without necessarily increasing the MOI.

While the ssDNA octopartite FBNSV has obviously not adopted this strategy at the within host level (see the multicellular lifestyle described above), a possible sorted transmission of distinct genome segments is suggested by Gilmer and colleagues $(39,40)$ for multipartite (+)ssRNA viruses. When reviewing the literature on the cell-to-cell and long distance within host movement of diantho-, bromo-, virga and bennyviruses the authors convincingly argue that these RNA genomes travel within the vascular system of their plant hosts under the form of nucleo-protein complexes (RNPs) and not mature assembled viral particles. The authors 
postulate that this matter of fact makes the RNA accessible within the moving RNPs and consider the possibility that specific RNA-RNA interactions between segments can elaborate a moving complex containing one copy of each. The movement of RNPs and the intersegment RNA-RNA interactions are well supported by empirical results. RNA-RNA intersegment interactions could have diverse roles in the viral cycle, however, and the direct demonstration that they tie the segments together during long distance within-host movement (Figure 1.3) is still lacking.

\subsection{Between-host cost}

As we just saw, the within-host cost can be at least partially alleviated either through a still hypothetical sorted transmission or because some viruses, such as the FBNSV, may adopt a multicellular way of life which dispenses them from the obligation of maintaining their genomic integrity at the single cell level. There is nevertheless an obligation to maintain genomic integrity at the individual host level: viruses need all genome segments (indispensable segments) in the same host individual in order to be able to successfully complete their lifecycle: replicate, colonize the host and successfully transmit the integral genome to another host. The between-host genomic integrity maintenance cost is obviously related to the transmission mechanisms. In plants, $>90 \%$ of the multipartite viral species are transmitted from-host-to-host by animal vectors (1) and must thus find a way to transfer all their genetic information.

An obvious solution could be through the massive inoculation of viral particles during vector transmission (Figure 1.5). This possibility is strongly contradicted by the currently 
available evidence: independently of their genomic architecture, nearly all viruses for which an empirical estimate exists undergo severe bottlenecks during host-to-host transmission (21). Only two multipartite viruses have been investigated in this respect: the tripartite (+)ssRNA cucumber mosaic virus (CMV; family Bromoviridae) and the octopartite ssDNA FBNSV. In both cases, the estimated effective bottleneck sizes were very low, ranging from one to 6 copies per segment ((41) for CMV; (36) for FBNSV), one to three orders of magnitude lower than the threshold beyond which mutlipartitism is theoretically favored $(32,36)$. It is true that effective sizes may differ from the number of particles actually transmitted. However, the FBNSV study used two methods: one based on genetic variant frequencies and estimating the genetic bottleneck, the other based on the success of infection and estimating the population bottleneck; both methods yielded very similar numbers.

Our admittedly very limited current knowledge thus leaves us with a still unresolved question as to how multipartite viruses manage to maintain their genomic integrity at this level. A potential critique of the laboratory-based estimations of the transmission bottlenecks is that they perhaps use unrealistically low numbers of vector individuals (aphids in these cases) per host plant, one to ten. Many aspects of multipartite virus ecology are currently unknown or at best understudied, and 'details' on aphid transmission are part of these aspects. The limited available evidence, however, does not suggest that a high density of vectors is the solution to this paradox: Schinghamer and colleagues found that only 10 individual aphids out of 447 investigated, belonging to three out of fourteen species, were able to transmit at least one out of nine virus diseases in a field population of faba bean (42). 
A bolder possibility is that perhaps all the segments do not need to colonize individual

plants concomitantly (Figure 1.7). We know for example that when the ssDNA nanoviruses successfully infect a host cell, their genetic information exits the capsid, moves to the host cell nucleus where the cell machinery polymerizes the second strand to produce dsDNA, which then associates with histone proteins to form a 'minichromosome' supporting both transcription and rolling circle replication (43). However, we do not know what happens if particles containing only some of the segments enter a host cell (Figure 1.8). The genetic information is likely similarly decapsidated, and if so, what is its fate in the absence of some of the segments? Does it wait for some signal under the form of a minichromosome? For how long can it stay in this state? Similar or at least analogous questions apply for genome segments of RNA multipartite 
genome segments of FBNSV have always been found to co-occur in field samples $(46,47)$, three segments, N, C and U4, are dispensable for infection to occur, at least under laboratory conditions $(46,47)$. It is however worth mentioning that the lack of at least some of these segments may have important phenotypic effects, e.g. in the absence of segment $\mathrm{N}$ successful infections are produced but aphid transmission becomes impossible (47). We inoculated one set of plants without segment $C$ and another set of plants without segment $U 4$. We then either (i) allowed different groups of aphids to feed on these two sets of plants and subsequently transferred both groups of aphids on naïve plants; or (ii) allowed the same aphids to acquire viral infections sequentially on the two sets of plants and then transferred them on naïve plants. In both cases we obtained successful infections of recipient plants containing all FBNSV segments. In an additional experiment aphids acquired first all segments but U4 from an incompletely infected plant, and then, three days later, U4 from an infected plant lacking $\mathrm{N}$. Using segment specific in situ hybridization, we could show that despite their sequential acquisition, $\mathrm{N}$ and $\mathrm{U} 4$ reunite and accumulate in the exact same intracellular membranous vesicles of the cells of the anterior midgut of the aphid vectors (Di Mattia J, Yvon M, Zeddam JL, Vernerey MS, Michalakis $\mathrm{Y}$ and Blanc S. In preparation). These experiments demonstrate that reassortment of incomplete infections may restore complete infections both very early within the gut of insect vectors upon cumulative storage of the segments sequentially acquired, or later within the plant after merging of incomplete infectious subsets of segments to restore the integral genome. It remains to be seen whether a similar outcome can be reached from incomplete inoculations which on their own are unable to establish successful infections, and to 
characterize the time-interval between inoculations allowing for the rescue of such incomplete infections (Figure 1.8).

\section{MULTICOMPONENT VIRAL SYSTEMS}

The previous sections outlined a number of issues imposed by their multipartite nature on these viruses, and some potential ways they found to accommodate them. Even though the situation is not identical, it is worth noting the analogies that exist between multipartite viruses and other multicomponent viral systems, such as segmented viruses, viral satellites or defective particles (48): the constraint to maintain genomic integrity and the possibility to regulate gene expression by differentially modifying the gene (segment) copy number. The solutions adopted by multipartite viruses could inspire research on these other viral entities, and reciprocally.

Segmented viruses do not face in principle the genomic integrity cost once viral particles have been formed. However, they must find a way to ensure that this indeed happens within individual host cells. This could happen either (i) through some specific sorting mechanism when packaging the distinct segments of the genome; or (ii) non-specifically packaging within each viral particle more segments than their genome consists of, analogous to a high $\mathrm{MOI}$ at the viral particle level.

Specific sorting mechanisms governing the packaging of the different genome segments have been described in several viruses. For example, Borodavka et al. (49) review the processes through which several dsRNA segmented viruses package their genomic segments in a specific sequence, which apparently ensures the incorporation of one copy of each segment in the viral particle. However, evidence accumulates that the (-)ssRNA Influenza A Virus (IAV) which also 
possesses specific packaging mechanisms (50), produces a large number of particles which fail to express all IAV genes, termed semi-infectious particles (or SIP $(10,51)$ ). They actually constitute the majority of IAV viral particles: a recent study reported that individual viral particle infections lead to the successful replication of all eight IAV segments in only $1.22 \%$ of all cases (52), and there is even variation among isolates in their propensity to produce such particles (see (53) for a review). As this review argues, SIPs may be generated by several mechanisms. Failure to package all eight segments has been reported to occur in up to $20 \%$ of viral particles (54). Failure to express the proteins of all eight segments could also be explained by post-inoculation segment loss during, e.g. segment trafficking within the cytoplasm or from the cytoplasm to the nucleus, or replication failure during the early stages of infection.

Whatever the mechanism responsible for SIP production, however, it is believed that the IAV manages to successfully infect its hosts through complementation resulting from multiple infections of single individual host cells (51-53), complementation being also the mechanism allowing the persistence of defective particles (55) and viral satellites (56). Observations on the (-)ssRNA Bunyavirales, e.g. Rift Valley fever virus (RVFV), reviewed by Wichgers Schreur et al. (57), also show that the majority of mature virions lack one or more genome segments, and that the genome segment ratio in mature virions departs from 1:1:1. Thus the existence of very specific packaging mechanisms does not per se alleviate the genomic integrity problem for viruses with genomes carried by more than one molecule. At least some of these viruses must resolve the issue through higher MOI.

It has been reported that at least one virus uses a "within-particles high MOI strategy" by packaging more segments in its viral particles than its genome consists of, the bi-segmented 
dsRNA infectious bursal disease virus (IBDV) of the family Birnaviridae (58): IBDV packages up to four segments in each particle. This results in an increased probability that at least one copy of each of its two segments will be carried by each particle, while at the same time some particles contain several copies of a given segment and other segments are missing: viral particles can be aneuploid, polyploid, and potentially both. None of the known multipartite viruses has adopted this packaging strategy: cases where more than one segments are packaged in the same particle exist, i.e. the RNA3 and RNA4 segments of bromoviruses (59) or RNA1 and RNA2 of dianthoviruses $(60,61)$, but it is always the same segments which are packaged together; the process is not random (62).

Finally, the possibility that the genomic integrity issue, at least at the within-host level, can be resolved by a multicellular way of life, as observed in the multipartite FBNSV (37), deserves further investigation in segmented viruses and also for accumulation/maintenance of satellites and defective particles. Host cells continuously traffic host functions, under the form of mRNA, proteins or even organelles (63-65). While it has long been evident that viruses exploit intra-host cell communication for cell-to-cell transmission, we do not know how frequently and how intensively they might use these avenues to circulate gene functions, thus adopting a multicellular way of life. This was shown to be the case in the multipartite FBNSV, but there is no a priori reason that such a functioning could be restricted to multipartite viruses; it could well occur e.g. in segmented viruses and even in monopartite.

Another feature put forward recently in multipartite viruses is the between segment variation in gene copy number, which further depends for a given virus on the host species (33, 34). It was suggested that this variation may lead to their ability to rapidly adapt their gene 
expression to the challenges imposed by differing host physiologies ((33, 34); M. Zwart and S.F.

Elena in review), and it was recently confirmed in the FBNSV that indeed DNA segment copy number variation is correlated with RNA expression: the quantitative variation at the gene level has a functional role in terms of gene expression (R. Gallet, J. Di Mattia, S. Ravel, R. Vitalis, Y. Michalakis and S. Blanc in preparation). The existence of SIPs and DIPs in many viruses result in within and between host variation of gene copy numbers and protein expression $(8,53,55,56)$, and it has been recently argued that this variation may at least in some cases provide advantages to the viruses ((53) for SIPs; $(55,66)$ for DIPs). It is thus possible to imagine that segmented viruses (and why not even some monopartite viruses) could function through SIP (or DIP for monopartite) production and a multicellular way of life as multipartite viruses within hosts, while resolving the between-host genomic integrity cost through the production of some viral particles containing all genomic segments (non-defective particles) and a relatively high MOI.

\section{RECAP ON CHALLENGES TO VIROLOGY PRINCIPLES POSED BY MULTIPARTITES AND OTHER} MULTICOMPONENT SYSTEMS

The existence of specific packaging mechanisms were at the basis of several foundational principles of virology: that one virus particle may be able to successfully infect one cell; that the viral genome travels between cells and hosts in a single transmission vehicle; that the viral replication cycle is "cell-autonomous", i.e. is completed within a cell and then reiterated in next contaminated cells. Multipartite viruses violate these principles. 

viruses: because they are multipartite the genetic information does not circulate in a single transmission vehicle. Instead, it is packaged in as many as eight different particles. The successful infection of a single cell cannot be achieved by a single viral particle, and the genomic integrity cost derives from these violations.

That the replication cycle is not cell-autonomous is a much more surprising violation, and one that allows to alleviate the genomic integrity cost, at least at the within-host level. It has been shown to occur in the octopartite nanovirus FBNSV (37). It is presently unclear whether this is a general feature of multipartite viruses, though there is no a priori reason to believe it should be restricted to just FBNSV, nanoviruses, or ssDNA viruses. It directly implies that the spatial unit of infection is not the individual host cell, but some larger level whose scale awaits further characterization.

We earlier mentioned the possibility that the between-host genomic integrity cost may be resolved if inoculation of a given host by all indispensable genomic segments does not need to be concomitant: if incomplete inoculations, where only some of the segments are passed to a host individual, may remain latent and be rescued by subsequent inoculations which complement them. We emphasize that this is still a hypothesis. But would it be proven to occur it would not only imply that the genomic integrity cost imposed by multipartitism is much weaker than initially anticipated. It would also imply that multipartite viruses can shuffle their genome through segment reassortments at a much larger scale than previously appreciated, even among individual viruses which do not co-occur on the same host individual, or even host population or perhaps host species - depending on vector behavior. It is unlikely that this 
potential, should its existence be proved, may have led to the evolution of multipartitism: considering a scenario where a rare multipartite mutant invades a monopartite population, because of its initial rareness the multipartite mutant could only benefit from its increased reassorting capacity too rarely: its genome segments would have a low probability to encounter other segments, and the very rare reassortments would need to be beneficial to constitute an advantage. This mechanism is thus unlikely to be at the origin of multipartitism. It can nevertheless provide the opportunity to multipartite viruses, once multipartitism established, to reshuffle their genome at a very high rate and e.g. generate host range variants (67) with obvious agricultural and economic consequences.

Multipartite viruses have been shown to be able to modify their gene copy number depending on the host species they infect through their genome formula modulation. In at least one of the viruses in which this phenomenon was observed, it was further shown that it has functional consequences since gene copy number variation is correlated with gene expression variation. These modifications occur 'immediately' upon a single passage from one host species to another, and they are not correlated with any mutations at the DNA sequence level (R. Gallet, J. Di Mattia, S. Ravel, R. Vitalis, Y. Michalakis and S. Blanc. in preparation). These observations lead to a number of important considerations:

First, they suggest that multipartite viruses may possess a mechanism allowing them to adjust their gene expression to their host species "instantly", upon a single transmission event. Because their aphid vectors are most often polyphagous, it may well be the case that they are often submitted to host species changes. This DNA mutation free gene expression adjustment mechanism could thus constitute an important advantage deriving directly from the 
multipartite nature of this viruses, a consideration supported by recent modeling results ( $M$.

Zwart and S.F. Elena in review). Even though monopartite viruses can also adapt to a challenging environment through copy number variation $(68,69)$, such adaptations require sequence mutations through gene duplication and, though relatively fast, are thus not immediate. This capacity to instantly modulate gene expression upon host species change, derived directly from multipartitism, may thus constitute an evolutionary advantage of this genomic architecture which under some circumstances may be able to overcome the genomic integrity cost - or whatever remains of it.

Second, the existence of the genome formula and its DNA-mutation free modification pose some formidable conceptual issues. What is the genome of a multipartite virus? Is it the concatenation of the DNA sequences of its genomic segments? Or is it instead the genome formula, i.e. the collection of all the copies of the different segments/genes? The latter would imply that the genome of a virus depends on its host species... Is gene copy number variation a genetic mutation or a manifestation of phenotypic plasticity in multipartite viruses? Nobody disputes that the adaptation of the monopartite poxviruses through gene copy number variation, termed 'genetic accordions' $(68,69)$ results from genetic mutations. Multipartite viruses react to host species switching through essentially the same mechanism, copy number variation; yet, if their genome is defined as the concatenation of the DNA sequences of their genomic segments, this adjustment should be considered as phenotypic plasticity since it does not result from a DNA sequence modification. To the extent that SIPs, and even DIPs, may not always represent junk for segmented viruses but functional explorations in gene copy number 
507

508

509

510

511

512

513

514

515

516

517

518

519

520

521

522

523

524

525

526

variation at least under some circumstances, as discussed earlier, these considerations may apply much more generally than one could think.

\section{DISCLOSURE STATEMENT}

The authors are not aware of any affiliations, memberships, funding, or financial holdings that might be perceived as affecting the objectivity of this review.

\section{ACKNOWLEDGMENTS}

We thank Rosemary Dorrington for clarifications on packaging of RNA segments of omegatetraviruses. This project was funded by the French national research funding agency (grants ANR-14-CE02-0014-01 and ANR-18-CE92-0028-01), the SPE department of INRA, and the IRD and CNRS research institutes.

\section{LITERATURE CITED}

1. Lucía-Sanz A, Manrubia S. 2017. Multipartite viruses: adaptive trick or evolutionary treat? npj Systems Biology and Applications. 3(1):

2. Sicard A, Michalakis Y, Gutiérrez S, Blanc S. 2016. The strange lifestyle of multipartite viruses. PLOS Pathogens. 12(11):e1005819

3. Liu W, Hajano J-U-D, Wang X. 2018. New insights on the transmission mechanism of tenuiviruses by their vector insects. Current Opinion in Virology. 33:13-17 
4. Dietzgen RG, Freitas-Astúa J, Chabi-Jesus C, Ramos-González PL, Goodin MM, et al. 2018. Chapter Five - Dichorhaviruses in their host plants and mite vectors. In Advances in Virus Research, ed P Palukaitis, MJ Roossinck. 102:119-48. Academic Press

5. Leastro MO, Kitajima EW, Silva MS, Resende RO, Freitas-Astúa J. 2018. Dissecting the subcellular Leprosis Virus C proteins. Front. Plant Sci. 9:

6. Hu Z, Li G, Li G, Yao Q, Chen K. 2013. Bombyx mori bidensovirus: The type species of the new genus Bidensovirus in the new family Bidnaviridae. Chin. Sci. Bull. 58(36):4528-32

7. Ladner JT, Wiley MR, Beitzel B, Auguste AJ, Dupuis AP, et al. 2016. A multicomponent animal virus isolated from mosquitoes. Cell Host \& Microbe. 20(3):357-67

8. Wichgers Schreur PJ, Kortekaas J. 2016. Single-molecule FISH reveals non-selective packaging of Rift Valley Fever Virus genome segments. PLOS Pathogens. 12(8):e1005800

9. Gokhale DV, Bald JG. 1987. Relationship between plant virus concentration and infectivity: a 'growth curve' model. Journal of Virological Methods. 18(4):225-32

10. Brooke CB, Ince WL, Wrammert J, Ahmed R, Wilson PC, et al. 2013. Most Influenza A virions fail to express at least one essential viral protein. Journal of Virology. 87(6):3155-62

11. Lister RM. 1966. Possible relationships of virus-specific products of tobacco rattle virus infections. Virology. 28(2):350-53

12. Van Kammen A. 1967. Purification and properties of the components of cowpea mosaic virus. Virology. 31(4):633-42

13. Chu PWG, Helms K. 1988. Novel virus-like particles containing circular single-stranded DNAs associated with subterranean clover stunt disease. Virology. 167(1):38-49

14. Hesketh EL, Saunders K, Fisher C, Potze J, Stanley J, et al. 2018. The $3.3 \AA ̊$ structure of a plant geminivirus using cryo-EM. Nature Communications. 9(1):2369 
15. Chou Y, Vafabakhsh R, Doğanay S, Gao Q, Ha T, Palese P. 2012. One influenza virus particle packages eight unique viral RNAs as shown by FISH analysis. Proceedings of the National Academy of Sciences. 109(23):9101-9106

16. Pressing J, Reanney DC. 1984. Divided genomes and intrinsic noise. J Mol Evol. 20(2):135-46

17. Nee S. 1987. The evolution of multicompartmental genomes in viruses. J Mol Evol. 25(4):277-81

18. Nee S, Maynard Smith J. 1990. The evolutionary biology of molecular parasites. Parasitology. 100(S1):S5-18

19. Szathmáry E. 1992. Natural selection and dynamical coexistence of defective and complementing virus segments. Journal of Theoretical Biology. 157(3):383-406

20. Szathmáry E. 1992. Viral sex, levels of selection, and the origin of life. Journal of Theoretical Biology. 159(1):99-109

21. Zwart MP, Elena SF. 2015. Matters of size: genetic bottlenecks in virus infection and their potential impact on evolution. Annual Review of Virology. 2(1):161-79

22. Zhang Y-J, Wu Z-X, Holme P, Yang K-C. 2019. Advantage of being multicomponent and spatial: multipartite viruses colonize structured populations with lower thresholds. Physical Review Letters. 123(13):138101

23. Varsani A, Lefeuvre P, Roumagnac P, Martin D. 2018. Notes on recombination and reassortment in multipartite/segmented viruses. Current Opinion in Virology. 33:156-66

24. Lefkowitz EJ, Adams, MJ, Davison, AJ, Siddell, SG, Simmonds, P, eds. 2015. Virus Taxonomy: The classification and nomenclature of viruses. The online 10th Report of the ICTV (EC 47, London, 2015). URL: https://talk.ictvonline.org/ictv-reports/ictv_online_report/

25. Lucía-Sanz A, Aguirre J, Manrubia S. 2018. Theoretical approaches to disclosing the emergence and adaptive advantages of multipartite viruses. Current Opinion in Virology. 33:89-95 
26. Chao L. 1991. Levels of selection, evolution of sex in RNA viruses, and the origin of life. Journal of Theoretical Biology. 153(2):229-46

27. Chao L. 1988. Evolution of sex in RNA viruses. Journal of Theoretical Biology. 133(1):99-112

28. Nee S. 1989. On the evolution of sex in RNA viruses. Journal of Theoretical Biology. 138(3):407-12

29. Ojosnegros S, García-Arriaza J, Escarmís C, Manrubia SC, Perales C, et al. 2011. Viral genome segmentation can result from a trade-off between genetic content and particle stability. PLOS Genetics. 7(3):e1001344

30. Garcia-Arriaza J, Manrubia SC, Toja M, Domingo E, Escarmis C. 2004. Evolutionary transition toward defective RNAs that are infectious by complementation. J. Virol. 78:11678-85

31. Valdano E, Manrubia S, Gómez S, Arenas A. 2019. Endemicity and prevalence of multipartite viruses under heterogeneous between-host transmission. PLOS Computational Biology. 15(3):e1006876

32. Iranzo J, Manrubia SC. 2012. Evolutionary dynamics of genome segmentation in multipartite viruses. Proc Biol Sci. 279(1743):3812-19

33. Sicard A, Yvon M, Timchenko T, Gronenborn B, Michalakis Y, et al. 2013. Gene copy number is differentially regulated in a multipartite virus. Nature Communications. 4:DOI: $10.1038 /$ ncomms3248

34. Wu B, Zwart MP, Sánchez-Navarro JA, Elena SF. 2017. Within-host evolution of segments ratio for the tripartite genome of Alfalfa Mosaic Virus. Scientific Reports. 7(1):5004

35. Hu Z, Zhang X, Liu W, Zhou Q, Zhang Q, et al. 2016. Genome segments accumulate with different frequencies in Bombyx mori bidensovirus. Journal of Basic Microbiology. 56(12):1338-43

36. Gallet R, Fabre F, Thébaud G, Sofonea MT, Sicard A, et al. 2018. Small bottleneck size in a highly multipartite virus during a complete infection cycle. Journal of Virology. 92(14):e00139-18 
37. Sicard A, Pirolles E, Gallet R, Vernerey M-S, Yvon M, et al. 2019. A multicellular way of life for a multipartite virus. elife. 8:e43599

38. Sanjuán R, Thoulouze M-I. 2019. Why viruses sometimes disperse in groups. Virus Evol. 5(1):

39. Dall'Ara M, Ratti C, Bouzoubaa SE, Gilmer D. 2016. Ins and outs of multipartite positive-strand RNA plant viruses: packaging versus systemic spread. Viruses. 8(8):228

40. Gilmer D, Ratti C, Michel F. 2018. Long-distance movement of helical multipartite phytoviruses: keep connected or die? Current Opinion in Virology. 33:120-28

41. Betancourt M, Fereres A, Fraile A, García-Arenal F. 2008. Estimation of the effective number of founders that initiate an infection after aphid transmission of a multipartite plant virus. Journal of Virology. 82(24):12416-21

42. Schwinghamer MW, Nicholas AH, Schilg MA. 2009. Three aphid vectors of faba bean (Vicia faba) viruses in northern New South Wales and occurrence of Acyrthosiphon pisum-transmitted isolates of Soybean dwarf virus. Australasian Plant Pathology. 38(3):262-69

43. Gronenborn B. 2004. Nanoviruses: genome organisation and protein function. Veterinary Microbiology. 98(2):103-9

44. Mossop DW, Francki RIB. 1978. Survival of a satellite RNA in vivo and its dependence on cucumber mosaic virus for replication. Virology. 86(2):562-66

45. Mossop DW, Francki RIB. 1979. The stability of satellite viral RNAs in vivo and in vitro. Virology. 94(2):243-53

46. Timchenko T. 2006. Infectivity of nanovirus DNAs: induction of disease by cloned genome components of Faba bean necrotic yellows virus. Journal of General Virology. 87(6):1735-43

47. Grigoras I, Vetten H-J, Commandeur U, Ziebell H, Gronenborn B, Timchenko T. 2018. Nanovirus DNA-N encodes a protein mandatory for aphid transmission. Virology. 522:281-91 
48. Michalakis Y, Blanc S. 2018. Editorial overview: Multicomponent viral systems. Current Opinion in Virology. 33:vi-ix

49. Borodavka A, Desselberger U, Patton JT. 2018. Genome packaging in multi-segmented dsRNA viruses: distinct mechanisms with similar outcomes. Current Opinion in Virology. 33:106-12

50. Hutchinson EC, von Kirchbach JC, Gog JR, Digard P. 2010. Genome packaging in influenza A virus. Journal of General Virology,. 91(2):313-28

51. Brooke CB. 2017. Population diversity and collective interactions during Influenza Virus infection. Journal of Virology. 91(22):e01164-17

52. Jacobs NT, Onuoha NO, Antia A, Steel J, Antia R, Lowen AC. 2019. Incomplete influenza A virus genomes occur frequently but are readily complemented during localized viral spread. Nat Commun. 10(1):1-17

53. Diefenbacher M, Sun J, Brooke CB. 2018. The parts are greater than the whole: the role of semiinfectious particles in influenza A virus biology. Current Opinion in Virology. 33:42-46

54. Nakatsu S, Sagara H, Sakai-Tagawa Y, Sugaya N, Noda T, Kawaoka Y. 2016. Complete and incomplete genome packaging of influenza $A$ and $B$ viruses. $m$ Bio. $7(5)$ :

55. Vignuzzi M, López CB. 2019. Defective viral genomes are key drivers of the virus-host interaction. Nature Microbiology. 4(7):1075

56. Gnanasekaran P, Chakraborty S. 2018. Biology of viral satellites and their role in pathogenesis. Current Opinion in Virology. 33:96-105

57. Wichgers Schreur PJ, Kormelink R, Kortekaas J. 2018. Genome packaging of the Bunyavirales. Current Opinion in Virology. 33:151-55

58. Luque D, Rivas G, Alfonso C, Carrascosa JL, Rodriguez JF, Caston JR. 2009. Infectious bursal disease virus is an icosahedral polyploid dsRNA virus. Proceedings of the National Academy of Sciences. 106(7):2148-52 
644

645

646

647

648

649

650

651

652

653

654

655

656

657

658

659

660

661

662

663

664

665

666

59. Chaturvedi S, Rao A. 2018. Molecular and biological factors regulating the genome packaging in single-strand positive-sense tripartite RNA plant viruses. Current Opinion in Virology. 33:113-19

60. Basnayake VR, Sit TL, Lommel SA. 2006. The genomic RNA packaging scheme of Red clover necrotic mosaic virus. Virology. 345(2):532-39

61. Basnayake VR, Sit TL, Lommel SA. 2009. The Red clover necrotic mosaic virus origin of assembly is delimited to the RNA-2 trans-activator. Virology. 384(1):169-78

62. Newburn LR, White KA. 2019. Trans-acting RNA-RNA interactions in segmented RNA viruses. Viruses. 11(8):751

63. Woith E, Fuhrmann G, Melzig MF. 2019. Extracellular vesicles-connecting kingdoms. International Journal of Molecular Sciences. 20(22):5695

64. Drab M, Stopar D, Kralj-Iglič V, Iglič A. 2019. Inception mechanisms of tunneling nanotubes. Cells. $8(6): 626$

65. Gill S, Catchpole R, Forterre P. 2019. Extracellular membrane vesicles in the three domains of life and beyond. FEMS Microbiol Rev. 43(3):273-303

66. Rezelj VV, Levi LI, Vignuzzi M. 2018. The defective component of viral populations. Current Opinion in Virology. 33:74-80

67. Pavithra BS, Govin K, Renuka HM, Krishnareddy M, Jalali S, et al. 2019. Characterization of cucumber mosaic virus infecting coleus (Plectranthus barbatus) in Karnataka. VirusDis. 30(3):40312

68. Elde NC, Child SJ, Eickbush MT, Kitzman JO, Rogers KS, et al. 2012. Poxviruses deploy genomic accordions to adapt rapidly against host antiviral defenses. Cell. 150(4):831-41

69. Bayer A, Brennan G, Geballe AP. 2018. Adaptation by copy number variation in monopartite viruses. Current Opinion in Virology. 33:7-12

667 
669 (reference number as in Literature Cited, annotation text):

6701 : important review on the evolution of multipartite viruses

6712 : important review on multipartite viruses with focus on different steps of their life cycle

672 10: Founding study revealing the problem of genome integrity in segmented viruses

673 21: reviews concepts and results on virus bottleneck sizes and MOI

674 23: reviews observations in recombination and reassortment in segmented and multipartite

675 viruses

676 29: only empirical paper exploring the potential advantages of multipartite variants

677 32: theoretical investigation of the parameter range favoring multipartitism depending on the

678 number of segments

679 33: first paper demonstrating the existence of the genome formula in multipartite viruses

680 37: first paper demonstrating a supra-cellular way of life for a multipartite virus

681 41: first paper estimating the bottleneck size in a multipartite virus 


\section{Caption figure 1}

A multipartite virus with 8 genome segments is used as an example for the illustration

1-When particles randomly enter cells at low MOI (thin arrows), each infected cell rarely receives the full set of segments. Complementation of distinct segments across cells allows the viral system to infect and maintain its genome integrity at a supra cellular level. This possibility has been experimentally demonstrated for FBNSV and is visible in the inset micrograph where two segments are FISH-labeled with distinct colours (37).

2-Each cell could randomly receive the full set of segments if entering at high $\mathrm{MOI}$ (thick arrows). This possibility has thus far not received empirical support (see text)

3-The full set of segments could be introduced into individual cells at low MOI if sorted cell-tocell transfer can operate. Such a specific sorting of the segments during cell-to-cell transfer has been discussed (40) but never fully demonstrated experimentally.

4-Once the host is infected, populations of multipartite viruses accumulate at specific frequencies for each segment (genome formula) that are host dependent. Here two distinct host species are represented in the upper and lower panels. It has been demonstrated in FBNSV that these distinct formulas correspond to different gene expression in the two hosts (R. Gallet, J. Di Mattia, S. Ravel, R. Vitalis, Y. Michalakis and S. Blanc in preparation). This ability to rapidly modify gene expression could represent an evolutionary advantage if host switches are frequent. 
5-Multipartite viruses could be transmitted between hosts at high $\mathrm{MOI}$, but no experimental data support this possibility

6-Sorted transmission of the segments could also occur at this level but this has not been shown in any case and it is not considered probable.

7-Current data indicate that transmission by insect vectors is at low $\mathrm{MOI}$ and so it is imaginable that distinct vector individuals could transmit incomplete sets of segments that would complement within the host (8) and initiate infection. 

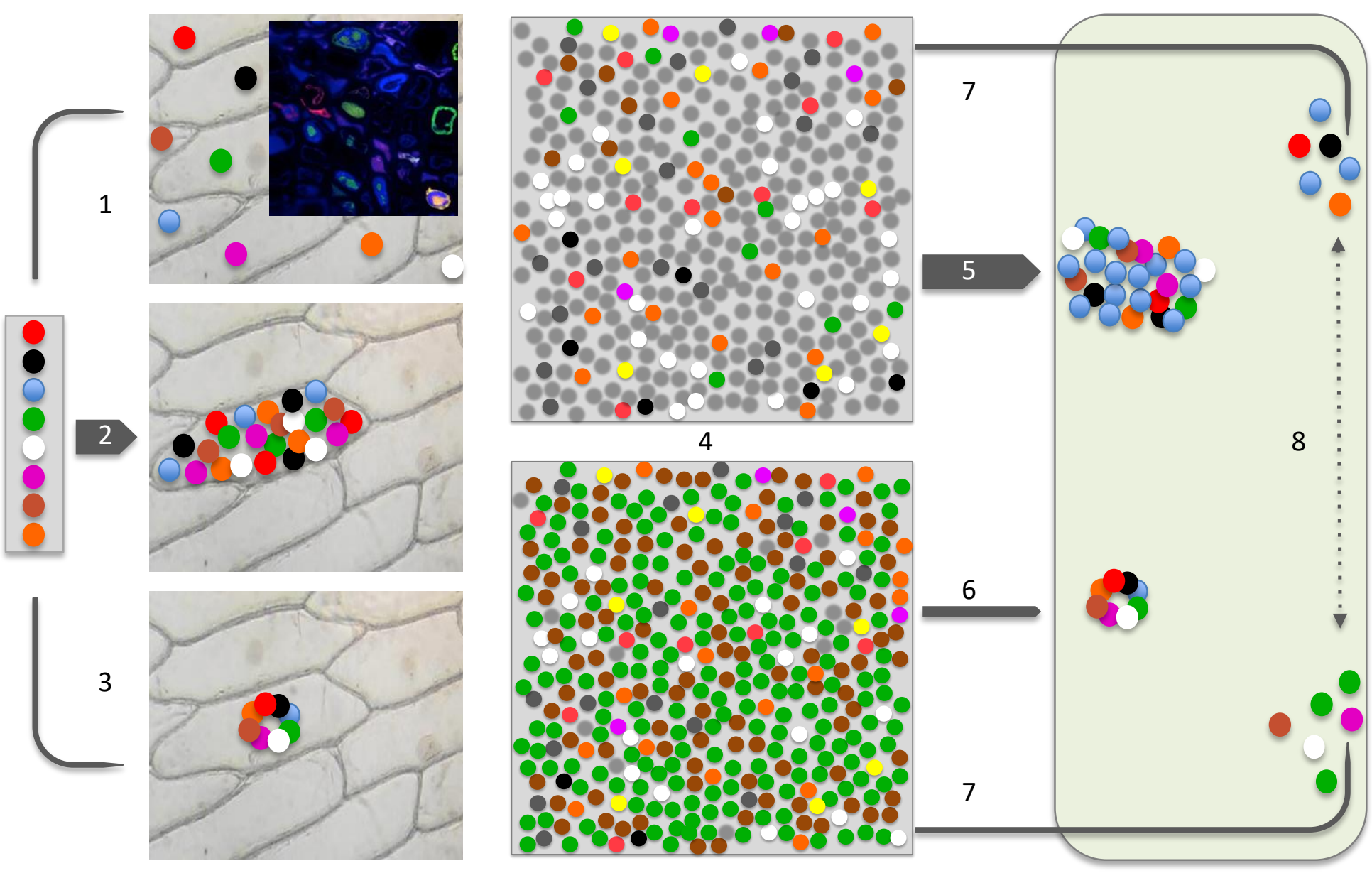


\section{Caption figure 2}

This figure is intended to use the data available at the time of preparation of this review to reveal the major trends in the distribution of the multipartite viral genome organization among types of host organisms and among the nature of the various nucleic acids composing the viral genomes. The numbers may not be exact for several reasons: i) packaging mechanisms have not been experimentally validated in many cases and may thus be deduced from relatedness with viral species where they were exprerimentally established, ii) some genera are unassigned to families, iii) when a family contains species in distinct categories, it is counted in each case, iv) the taxonomy is not fixed and so the number of genera and families is continuously changing over the years. This figure represents our interpretation of information from the literature and from ViralZone (https://viralzone.expasy.org/). In each diagram the numbers represent numbers of genera and the numbers in parentheses numbers of families. A: variation in genomic architecture across different types of hosts. The cells in Animals overlapping with those in plants/fungi correspond to plant viruses replicating in their animal vectors. When the hosts are not identified, the corresponding genera and families are simply not counted. B: variation in genomic architecture across different types of nucleic acid; C: variation in nucleic acid across different types of hosts. The cells in Animals overlapping with those in plants/fungi correspond to plant viruses replicating in their animal vectors. When the hosts are not identified, the corresponding genera and families are simply not counted. 


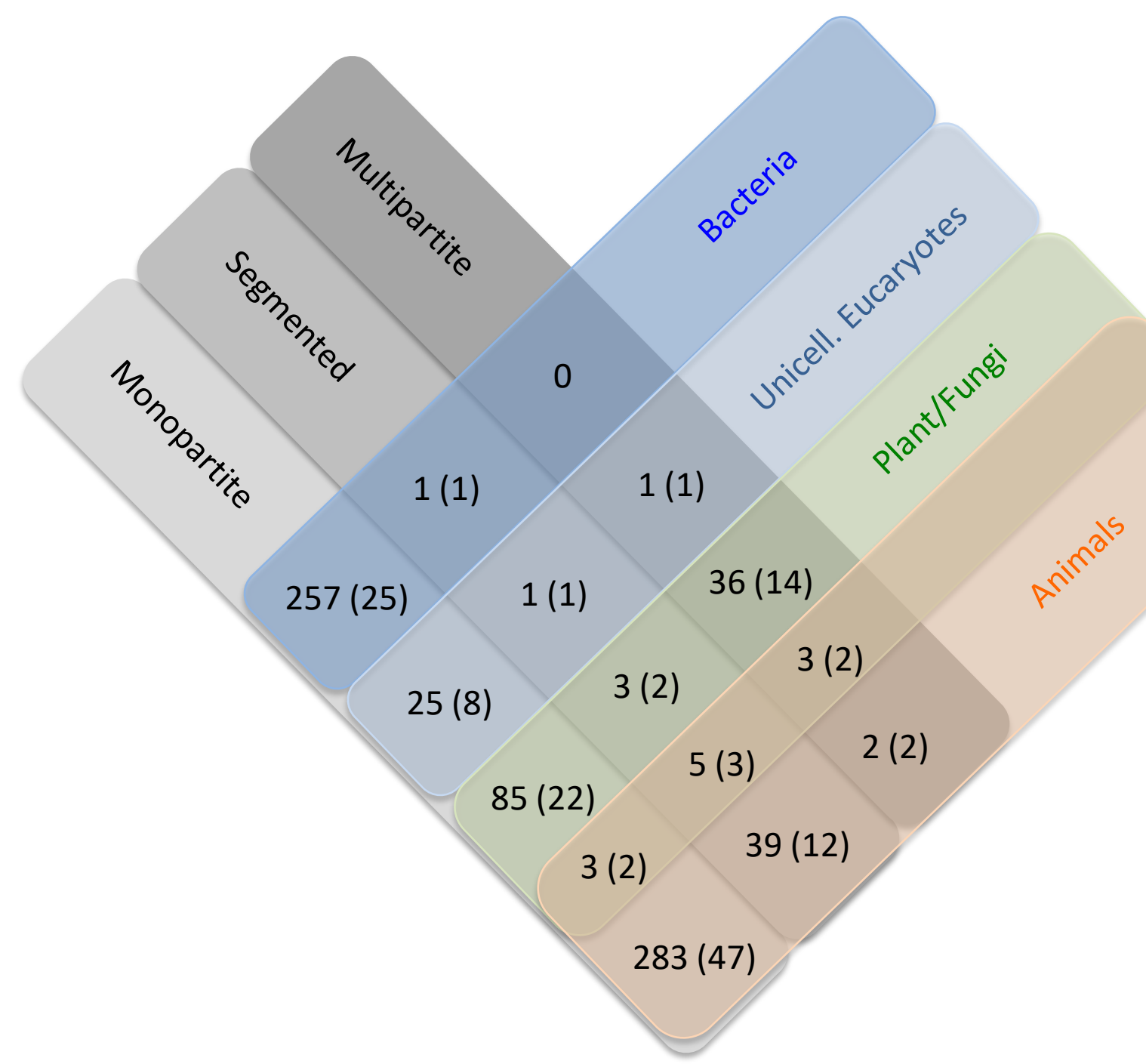




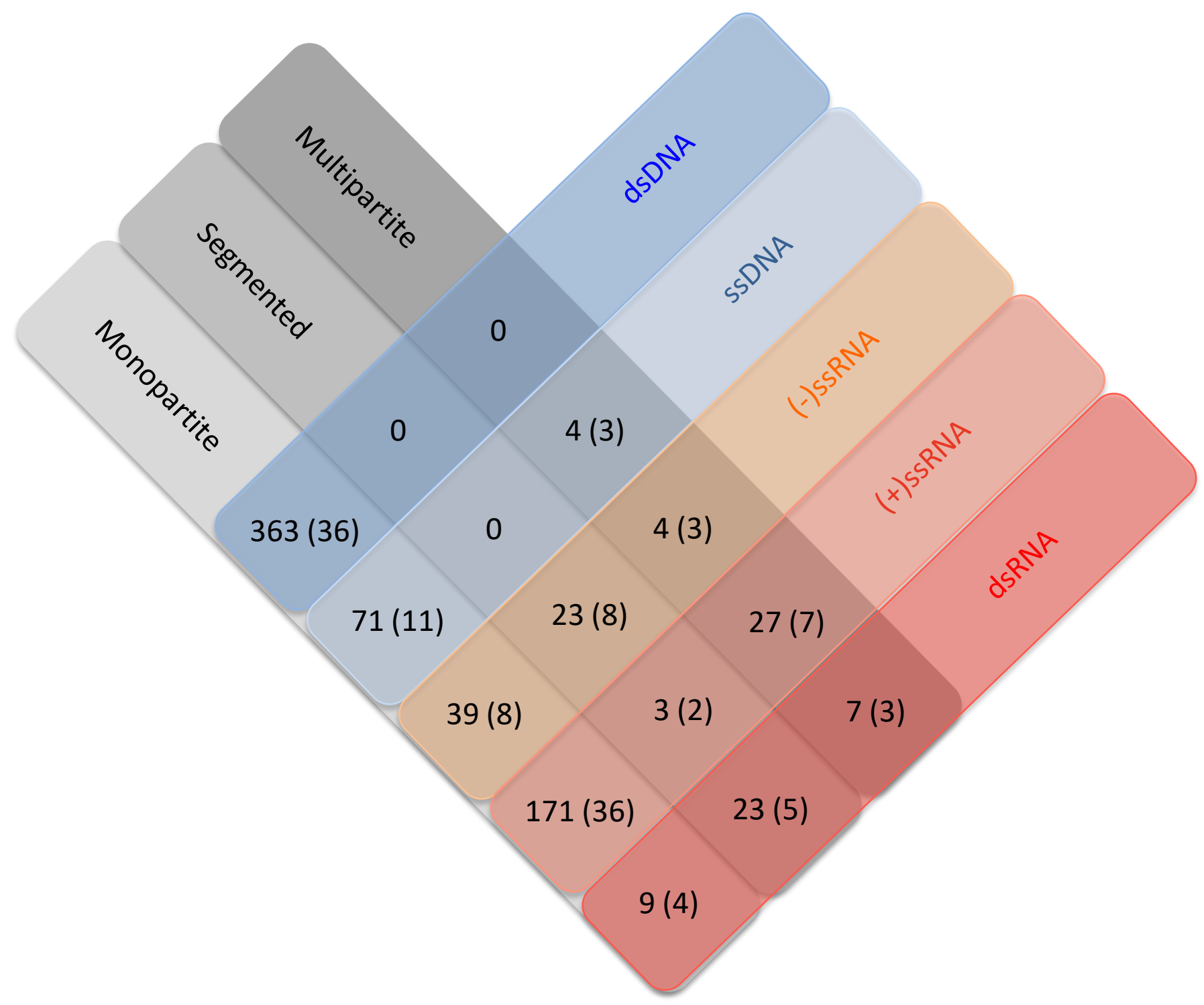


C

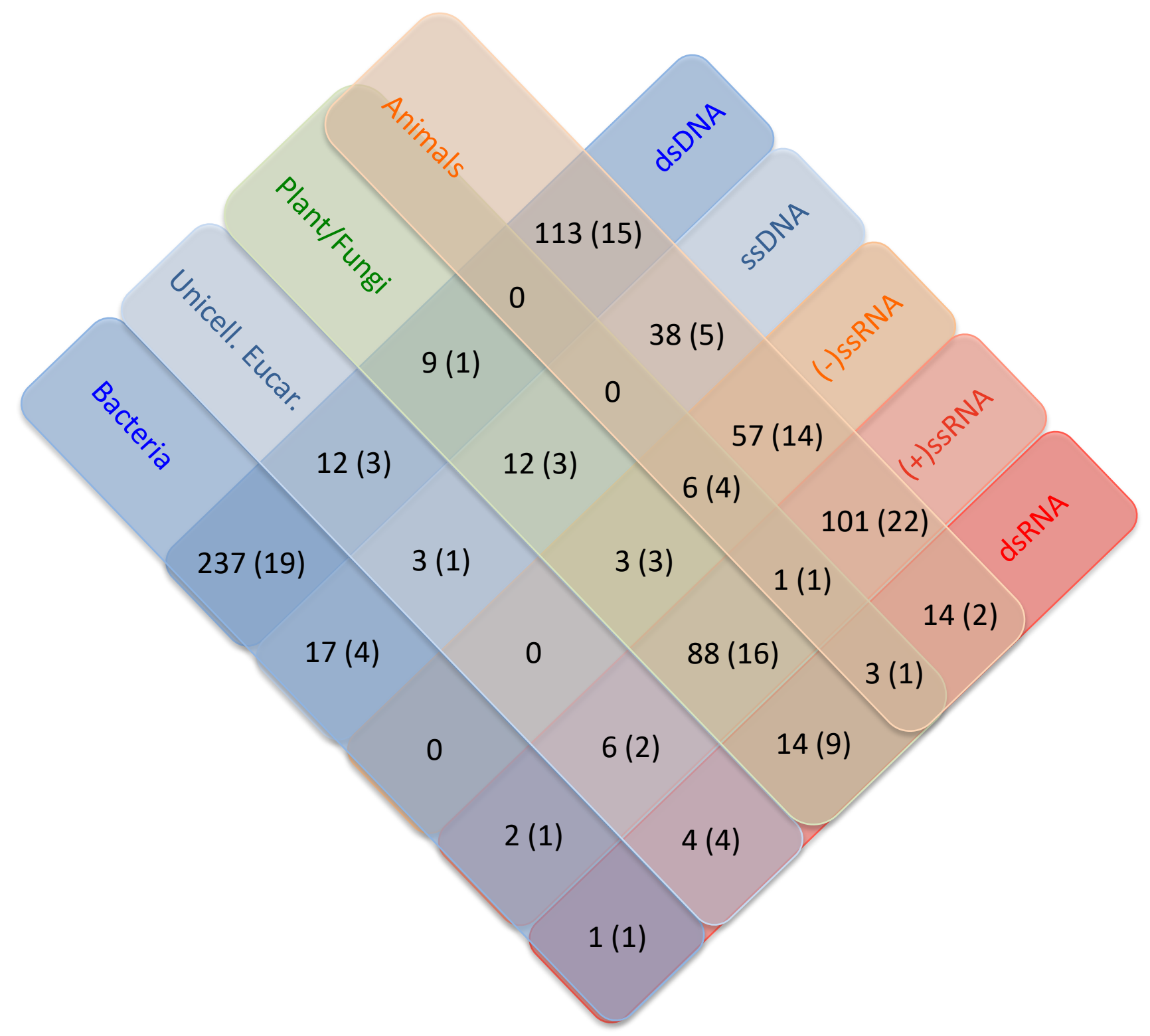




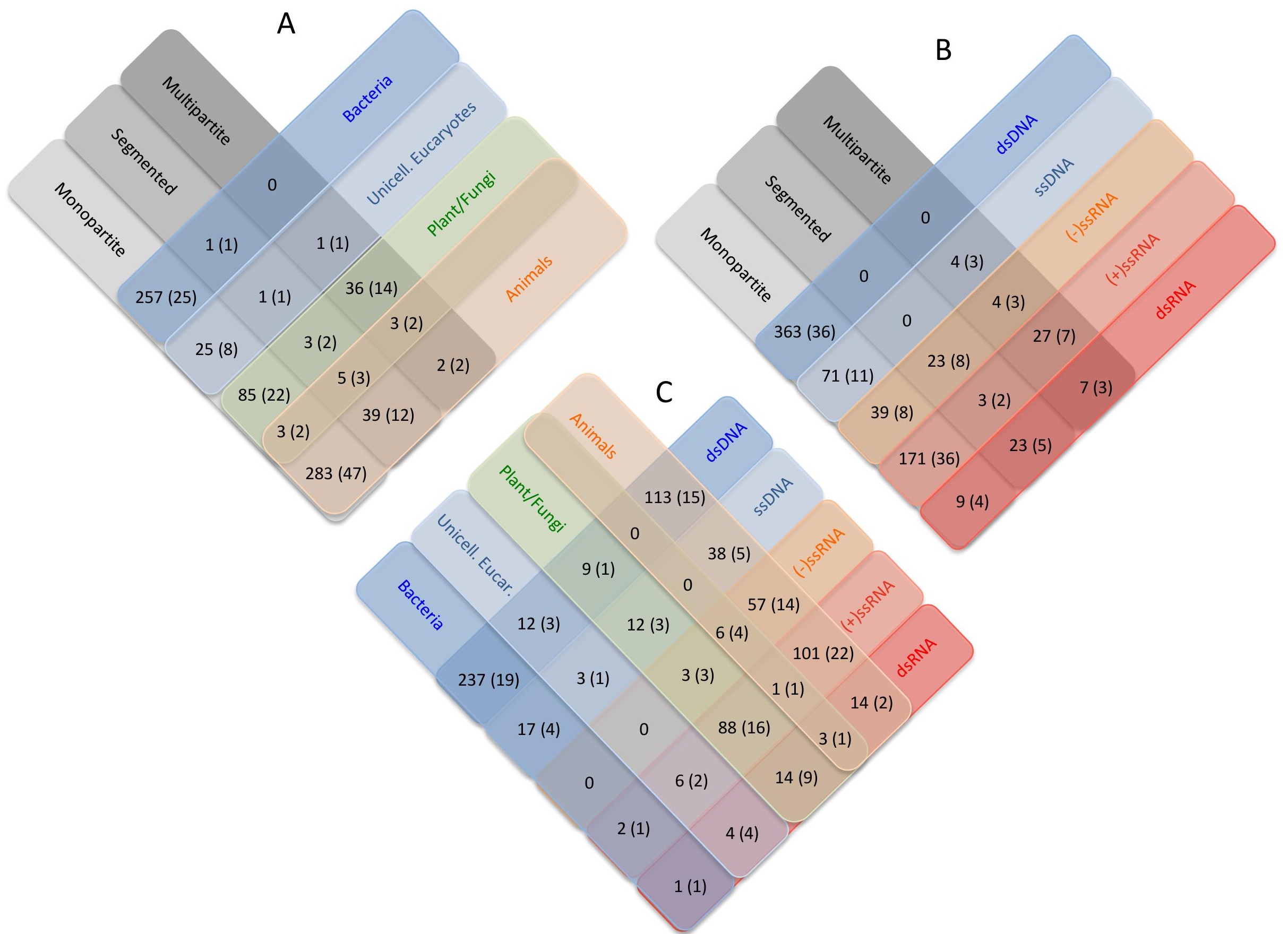

